\title{
APRENDIZAJE COLABORATIVO CON TIC Y LAS EXCEPCIONES Y LIMITACIONES AL DERECHO DE AUTOR: COLOMBIA
}

\author{
MARCELA PALACIO PUERTA*
}

\section{RESUMEN}

La tecnología y en particular la internet han empezado a influenciar y modelar la educación. Debido a la existencia de la tecnología se puede pensar en un nuevo modelo educativo, con nuevas metodologías tales como el aprendizaje colaborativo. Colombia es uno de los países que están buscando transformar la educación a través de la tecnología; no obstante, el presente artículo, con un método hermenéutico, concluye que su ley de derechos de autor parece no estar adaptándose a dicha realidad, especialmente en el caso de excepciones y limitaciones.

Palabras clave: educación, tecnología, excepciones y limitaciones, derechos de autor, aprendizaje colaborativo.

\section{COLLABORATIVE LEARNING WITH ICT AND EXCEPTIONS \\ AND LIMITATIONS TO COPYRIGHT: COLOMBIA}

\section{ABSTRACT}

Technology, and especially the Internet, has influenced and shaped education. It is due to the existence of technology that one can think about a new educational model, with new educational methodologies, such as collaborative methodology. Colombia is one of the countries that are trying to transform education through

\footnotetext{
* Docente-investigadora, Universidad Santo Tomás (seccional Tunja). Doctora en derecho de la American University Washington College of Law. Bogotá (Colombia). Contacto: marcela. palaciop@usantoto.edu.co. Fecha de recepción: 23 de abril de 2020. Fecha de aceptación: 19 de febrero de 2020. Para citar el artículo: Palacio Puerta, Marcela. Aprendizaje colaborativo con TIC y las excepciones y limitaciones al derecho de autor: Colombia. Revista La Propiedad Inmaterial n.o 29, Universidad Externado de Colombia, enero-junio, 2020, pp. 117-136. DoI: https://doi.org/10.18601/16571959.n29.05.
} 
technology. Nonetheless, this paper while applying a hermeneutic analysis concludes that Colombia's copyright law does not seem to be adapting to that reality, especially in the case of exceptions and limitations.

Keywords: education, technology, limitations and exceptions, copyright, collaborative learning.

\section{INTRODUCCIÓN}

La educación y los derechos de autor han estado unidos desde un comienzo. Por esto, la legislación de derechos de autor ha proferido unas limitaciones y excepciones en favor de la educación. No obstante, la humanidad ha evolucionado y el modelo educativo y las necesidades de aprendizaje bajo los cuales se habían pensado dichas excepciones y limitaciones han variado dada la importancia de la tecnología en la vida actual.

La influencia de la tecnología en la educación ha permitido y buscado que el modelo educativo cambie en el sentido en que los roles tradicionales de los intervinientes se modifiquen y, entre otras cosas, se enseñe y aprenda a través de la colaboración. Estos componentes, que giran en torno a un nuevo modelo educativo y nuevas oportunidades de aprendizaje, gracias a la existencia de tecnologías como PC, tabletas e internet, modifican el escenario en el que el derecho de autor y la educación solían encontrarse creando nuevas fricciones. Aunque la literatura existente ha abarcado el tema de las diferentes fricciones que causa incorporar las tecnologías en el proceso educativo, incluyendo el aprendizaje colaborativo, en relación con la regulación autoral, esta se ha enfocado principalmente respecto de las figuras de la responsabilidad de los proveedores de servicio de internet y las medidas tecnológicas de protección ${ }^{1}$.

De esta manera, el presente artículo, con un método jurídico-dogmático y hermenéutico, entra a explicar las fricciones que existen entre lo que sería un modelo educativo de aprendizaje colaborativo con tecnologías de la información y la comunicación (TIC) y las mencionadas excepciones y limitaciones creadas para la educación, para concluir que la ley de derecho de autor colombiana debe ser modificada para permitir la evolución de dicho modelo educativo, especialmente por tres inconvenientes que se presentan al momento de intentar aplicar las excepciones y limitaciones a dicho tipo de aprendizaje: estas son (1) dificultad para aplicar las excepciones y limitaciones al mundo digital; (2) dificultades para aplicar las excepciones y limitaciones a otros actores diferentes a los docentes; y (3) dificultades para aplicar las excepciones y limitaciones a las actividades o metodologías colaborativas. 


\section{LAS EXCEPCIONES Y LIMITACIONES PARA}

\section{LA EDUCACIÓN EN EL DERECHO DE AUTOR}

Desde los inicios del derecho de autor, éste se ha conectado con la educación. Lo anterior se debe a que casi todos los materiales utilizados en la enseñanza son obras protegidas por el derecho de autor ${ }^{2}$, y en consecuencia este último tiene la habilidad de determinar cómo las personas aprenden ${ }^{3}$. Por esta razón desde los inicios del derecho de autor tanto en el ámbito nacional ${ }^{4}$ como en el internacional se estableció que la protección autoral debería estar sometida a ciertos límites para proteger o promover ciertos intereses públicos como la educación 5 .

En Colombia tanto la legislación doméstica, Ley 23 de 1982 con sus leyes modificatorias, como la regional, Decisión Andina 351, establecieron ciertas limitaciones al monopolio entregado por el derecho de autor a sus titulares en aras de promover intereses públicos tales como la educación, la información y el acceso a la cultura permitiendo ciertas utilizaciones de una obra protegida por parte de un tercero sin la necesidad de obtener autorización ni de realizar un pago $^{6}$. Dicho sistema de limitaciones y excepciones se ha conocido como de numerus clausus, consistente, como se dijo, en una lista exhaustiva de excepciones y limitaciones que debe interpretarse de manera restringida y no acepta aplicación analógica, diferenciándose de otros sistemas utilizados en el campo comparado, como el "fair use" o "fair dealing".

Otras características de las excepciones y limitaciones establecidas por el derecho de autor colombiano es que éstas siempre deben estar enmarcadas dentro de la regla de los tres pasos, ya sea durante su creación o su aplicación. La llamada regla de los tres pasos proscribe que las excepciones y limitaciones deben responder a tres circunstancias (a) que se trate de un caso especial y taxativamente establecido en la ley; (b) que no se atente contra la normal explotación de la obra; y (c) que tal limitación no cause perjuicio injustificado a los legítimos intereses del titular de los derechos.

Dicha regla tuvo origen en el ámbito internacional en la Conferencia de Estocolmo sobre el Convenio de Berna como reacción al derecho general de reproducción

2 Wahid Ratnaria, The Fairness of 'Stealing' Knowledge for Education, J. Int'l Com. L. \& Tech., vol. 6, 2011, p. 87.

3 See Kapczynski Amy, Access to Knowledge: A Conceptual Genealogy, in Access to Knowledge, New York, Zone Books, 2010, p. 24.

4 Dutfield Graham \& Suthersanen Uma, Global Intellectual Property Law, Northhampton, Edward Elgar, 2008, p. 55.

5 Ricketson Sam, Ginsburg Jane C., International Copyright and Neighbouring Rights: The Berne Convention and Beyond, Oxford, Oxford University Press, 2nd ed., vol. I, 2006, p. 756.

6 Dirección Nacional de Derecho de Autor, Generalidades del Derecho de Autor y Derechos Conexos. Autorización previa y expresa-limitaciones (n. d.), radicación n. ${ }^{\circ}$ 1-2014-27565 p. 4, disponible en http://200.91.225.128/Intrane1/desarrollo/CONCEPTOSWEB/arch_conceptos/1-2014-27565.pdf.

7 Monroy Juan Carlos, Estudio Sobre las limitaciones o excepciones al derecho de autor y los derechos conexos en beneficio de las actividades educativas y de investigación en América Latina y el Caribe, Ginebra, Comité Permanente de Derecho de Autor y Derechos Conexos 2009, p. 35, disponible en http://www.wipo.int/meetings/en/doc_details.jsp?doc_id=130303. 
que se acababa de reconocer ${ }^{8}$, buscando instaurar una guía sobre el alcance y contenido que podrían tener las excepciones y limitaciones respecto de dicho derecho promulgadas por el legislador dentro de un país miembro. Consecutivamente, la regla de los tres pasos se incluyó en los tratados internacionales posteriores sobre la materia, pero esta vez con un sentido más amplio, ya que abarcó dentro de su ámbito de cobertura los demás derechos exclusivos ${ }^{9}$ como en el artículo $10{ }^{\circ}$ del Tratado de la OMPI sobre Derechos de Autor (TODA), el artículo 16 de Tratado de la OMPI sobre Interpretación o Ejecución y Fonogramas (TOIEF) y el artículo 13 del Acuerdo de Propiedad Intelectual Relacionados con el Comercio (ADPIC). En el espacio regional, la regla de los tres pasos fue incorporada por el legislador andino en la Decisión 351, artículo 21.

Además, en Colombia las excepciones y limitaciones durante su aplicación se encuentran guiadas por el principio de uso honrado establecido en el artículo $3 .^{\circ}$ de la Decisión Andina $351^{10}$, el cual presenta gran semejanza con la regla de los tres pasos ya que hace referencia a aquellos actos que "[...] no interfieren con la explotación normal de la obra ni causan un perjuicio irrazonable a los intereses legítimos del autor". De esta manera, la Decisión 351 exige que aunque la excepción y limitación se haya promulgado en cumplimiento de la regla de los tres pasos, en su aplicación también se cumpla con estos parámetros.

Respecto de las excepciones y limitaciones para promover la educación, la Ley 23 de 1982 y la Decisión 351 establecen una serie de casos en los que se podrá utilizar la obra sin necesidad de autorización ni pago con el fin de permitir actividades propias de la enseñanza. Su función en la educación es evidente: por ejemplo, y solo por mencionar algunos, el artículo 22 (b) de la Decisión 351 establece una excepción y limitación al derecho de reproducción en favor de las instituciones educativas para la enseñanza o evaluación. Esta excepción permite a los profesores realizar copias de calidad de materiales pertinentes sobre temas tratados en las clases y compartirlas con sus alumnos o utilizarlos con fines de evaluación, siempre que se realice dentro de ciertos límites y respetando determinados requisitos que la ley establece, tales como indicar el nombre del autor de la obra y la fuente, que se haga conforme a los usos honrados y en la medida justificada por el fin que se persiga. Es así como esta limitación permite realizar una práctica que es común en el ambiente educativo donde los profesores complementan sus lecciones con materiales pertenecientes a terceros sin la necesidad de tener que adquirir una licencia.

8 Xalabarder Raquel, Estudio sobre las limitaciones y excepciones del derecho de autor para las actividades educativas en América del Norte, Europa, los países Cáucaso, Asia Central e Israel, Ginebra, Comité Permanente de Derecho de Autor y Derechos Conexos,2009, p. 24, disponible en http://www.wipo.int/edocs/mdocs/copyright/es/sccr_19/sccr_19_8.pdf.

$9 \mathrm{Id}$. at 25.

10 Dirección Nacional de Derecho de Autor, Concepto competencia DNDA (n. d.), radicación n. ${ }^{\circ}$ 1-2016.100918, p. 10, disponible en http://200.91.225.128/Intrane1/desarrollo/ CONCEPTOSWEB/arch_conceptos/1-2016-100918.pdf. 
La excepción para la ilustración en la educación establecida en el artículo 32 de la Ley 23 del 82 permite utilizar obras protegidas literarias o artísticas o partes de ellas, a título de ilustración en la enseñanza por diferentes medios como las publicaciones, emisiones de radiodifusión o grabaciones sonoras o visuales o comunicar con propósitos de enseñanza la obra radiodifundida siempre que se cumplan con ciertos requisitos como mencionar el nombre del autor y el título de la obra, se haga $\sin$ fines de lucro y en la medida justificada por el fin propuesto. De igual manera, el artículo 40 de la Ley 23 de 1982 protege el derecho a tomar notas de la clase y que van dirigidas a que el estudiante pueda construir su material de estudio; y los artículos 149 y 164 de la Ley 23 de 1982 permiten la representación o ejecución pública de obras protegidas dentro de las instituciones educativas, una vez más, siempre que se respeten ciertos límites.

Otras excepciones que no fueron propiamente establecidas como una excepción para la educación pero que cumplen un papel importante para ésta son la excepción de copia privada y la excepción de bibliotecas. La excepción de copia privada, establecida por el artículo 37 de la Ley 23 de 1982, es importante para la educación, ya que representa el acceso a la información, la educación y la cultura, y también representa un uso legal de las tecnologías ${ }^{11}$. Esta excepción permite a cualquier interesado hacer una reproducción de una obra literaria o científica, por cualquier medio, sin fines de lucro. Por otro lado, la excepción de uso privado, establecida en el artículo 44 de la Ley 23 de 1982, permite la utilización sin ánimo de lucro de las obras científicas, literarias y artísticas en un domicilio privado.

Finalmente, las excepciones en favor de las bibliotecas se establecen en el artículo 22 (c) de la Decisión 351 y en el artículo 38 de la Ley 23 de 1982. Bajo la legislación colombiana, estas excepciones permiten a las bibliotecas reproducir una obra en un solo ejemplar con tres fines específicos (1) para preservar el original y sustituirlo en caso de pérdida o destrucción; (2) para reemplazar las existencias permanentes de otra biblioteca en caso de pérdida o destrucción; y (3) para el uso de sus lectores.

Como se mencionó, estas excepciones y limitaciones fueron pensadas para promover el interés público de la educación; no obstante, dada la antigüedad de la regulación en la materia, dichas excepciones responden a las realidades de la enseñanza y las necesidades de ésta de los años 1982 y 1993. En el 2018, con la Ley 1915, se intentó realizar una modificación y actualización de la ley de derecho de autor para adaptarla al mundo digital, se abordaron temas novedosos como la protección de reproducciones temporales y protección contra la elusión a medidas tecnológicas de protección. No obstante, se dejó por fuera de la legislación cualquier referencia a excepciones y limitaciones para una educación fundamentada en el uso de las tecnologías, incluso para algo tan básico como la educación a distancia.

11 Dirección Nacional de Derecho de Autor, Concepto, limitaciones y excepciones en la legislación colombiana, abril 9, 2010, radicación n. ${ }^{\circ}$ 1-2010-7340, pp. 3-4, disponible en http://200.91.225.128/Intrane1/desarrollo/CONCEPTOSwEB/arch_conceptos/1-2010-7340.pdf. 
Por lo tanto, las excepciones hoy existentes están dispuestas para realidades propias de antes del auge de la internet y su influencia en diversas áreas de la vida humana como lo es la educación.

\section{EL NUEVO PAPEL DE LA INTERNET EN EDUCACIÓN}

Las nuevas tecnologías, y en especial la internet, han influido cada aspecto de la vida humana, y, por supuesto, la educación no ha sido ajena a este fenómeno. Hoy en día diversos países alrededor del mundo han optado por incorporar las TIC a su modelo educativo, buscando tener como resultado una transformación de este, como se explicará más adelante. Por ejemplo, en una encuesta de la Unesco a 38 países de la región latina y del Caribe, el $82 \%$ de los encuestados afirma tener ya sea una política pública, un plan o una institución encargada de fomentar la incorporación de las tecnologías en la educación ${ }^{12}$.

Cuando se habla de incorporar las tecnologías en educación se hace referencia a ir más allá de simplemente reemplazar una máquina de escribir por un PC o materiales impresos por archivos digitales ${ }^{13}$. Se trata de convertir la tecnología en el elemento central del proceso de aprendizaje y, por lo tanto, transformar dicho proceso. En un modelo educativo transformado por la tecnología, el profesor deja su papel de fuente proveedora de conocimiento para convertirse en un facilitador o coaprendiz y el estudiante abandona su papel pasivo y se vuelve creador de conocimiento a través de la colaboración ${ }^{14}$. De igual manera, lleva al desarrollo de nuevas metodologías y al uso de nuevos tipos de contenidos ${ }^{15}$.

Dentro de estas nuevas metodologías se pueden encontrar y se hace énfasis en la metodología colaborativa o aprendizaje colaborativo. Instaurar dicho aprendizaje colaborativo es posible gracias, entre otros aspectos, al auge de herramientas Web 2.0, con las cuales cualquier persona puede acceder a conocimiento pero también comunicarlo o compartirlo y colaborar con los demás participantes interactivamente. Ejemplos de estas herramientas son los blogs, trinos, plataformas de compartir video, entre otros. Una de las herramientas Web 2.0 más populares actualmente es YouTube ${ }^{16}$. Este nuevo escenario que permite la red 2.0 invita o de cierta forma exige que los intervinientes de la red asuman un papel activo donde éstos interaccionen para editar, colaborar, crear, modificar comunicar y compartir

12 Unesco, Uso de TIC en educación en América Latina y el Caribe, Montreal, Unesco, 2012, p. 7, disponible en https://unesdoc.unesco.org/ark:/48223/pf0000219369.

13 Unesco, Technologies for Education, Unesco, París, 2002, p. 8-9, disponible en http:// unesdoc.unesco.org/images/0011/001191/119129e.pdf.

14 Anderson Jonathan, Unesco Bangkok, ICT Transforming Education: a Regional Guide, Unesco, Tailandia, 2010, 6 tbl. 1.1; Unesco, supra note 14, at 14 .

15 Kalaš Ivan et al., Unesco, ICT in Primary Education, Unesco, Rusia, 2012, p. 22 disponible en http://iite.unesco.org/pics/publications/en/files/3214707.pdf.

16 Light Daniel \& Polin Deborah Keisch, edC Center for Children and Technology, Integrating Web 2.0 Tools into the Classroom: Changing the Culture of Learning, New York, Intel, 2010, p. 7 disponible en http://cct.edc.org/sites/cct.edc.org/files/publications/Integrating\%20 Web2.0.pdf. 
conocimiento ${ }^{17}$. Por ejemplo, gracias a la colaboración y a tecnologías Web 2.0 Wikipedia consiguió convertirse en la enciclopedia más grande del mundo.

En la educación con TIC, la metodología colaborativa se presenta dentro del salón de clase pero también por fuera de este. En relación con las actividades dentro del salón de clases, esta metodología busca instaurar un modelo en que los profesores y los estudiantes trabajen conjuntamente en proyectos e investigaciones que los lleven hacia la creación de nuevo conocimiento a partir de la interacción y el trabajo transformativo sobre el conocimiento preexistente ${ }^{18}$.

En relación con actividades fuera del salón de clase, la colaboración busca la creación de comunidades de aprendizaje donde gracias a las tecnologías, profesores y estudiantes se comunican con expertos a través del mundo e intercambian fuentes, materiales de clases, datos, opiniones, y realizan proyectos conjuntos. Dicha metodología es importante para la sociedad actual, en que los empleadores buscan personas con la capacidad de trabajar en equipo y $\mathrm{crear}^{19}$. De esta manera, como lo afirman Solano y Gutiérrez, "[...] la web nos exige que editemos información y construyamos colaborativamente el conocimiento" 20 , y esta realidad es o debe ser llevada al aprendizaje. Actualmente existen varios ejemplos de comunidades de aprendizaje o de herramientas que promueven la colaboración en la educación, como Edublogs, donde se permite a los investigadores y profesores intercambiar datos, fuentes, experiencias, entre otras, con la comunidad interesada ${ }^{21}$.

Colombia no ha sido ajena a la tendencia de buscar una transformación del proceso de aprendizaje fundamentado en las TIC. El país ha invertido grandes sumas de dinero para lograr una incorporación de la tecnología en la educación. Por ejemplo, en el periodo de agosto de 2010 a julio de 2014 el Ministerio de Tecnologías de la Información y las Comunicaciones invirtió la suma de $\$ 611.048$ millones de pesos en la entrega de terminales de cómputo para sedes educativas, bibliotecas y casas de la cultura ${ }^{22}$. Actualmente se siguen realizando inversiones de este tipo. Por ejemplo, durante el periodo de junio de 2016 a mayo de 2017 el Ministerio de Tecnologías de la Información y las Comunicaciones invirtió $\$ 83.000$ millones de pesos dirigidos a brindar acceso a TIC, formación educativa y sostenibilidad ambiental a sedes educativas seleccionadas ${ }^{23}$.

17 Solano I. \& GutıÉrRez I. Herramientas para la colaboración en la enseñanza superior: wikis y blogs, En Enseñanza Universitaria en el marco del Espacio Europeo de Educación Superior, 2007, p. 6-8 disponible en https://digitum.um.es/digitum/bitstream/10201/13414/1/Wikis_Blogs.pdf.

18 Kozma Robert B. \& Shafika Isaacs, Unesco, Transforming education: the power of ICT policies, Francia, Unesco, 2011, p. 22 disponible en http://unesdoc.unesco.org/images/0021/002 118/211842e.pdf.

19 Unesco, supra note 14 , at 36.

20 Solano I. \& Gutiérrez I. supra note 18, at 6-8.

21 Palacio Puerta, supra note 2.

22 Ministerio de Tecnologías de la Información y las Comunicaciones, Informe de Rendición de Cuentas, 2014, p. 40, disponible en http://www.mintic.gov.co/portal/604/articles-4323_recurso_1.pdf. (stating the buged used for handing computers and tablets).

23 Ministerio de Tecnologías de la Información y las Comunicaciones, Informe de Gestión al Congreso de la República 2017 Sector Tic, 2017, p. 26, disponible en https://www. mintic.gov.co/portal/604/articles-54877_doc_pdf.pdf. 
Inversiones de esta clase hacen parte de diversos programas que ha venido desarrollando el Ministerio de Tecnologías de la Información y las Comunicaciones al igual que el Ministerio de Educación Nacional con el propósito de incorporar las TIC en la educación, como el Programa de Uso de Nuevas Tecnologías para el Desarrollo de Competencias, un programa con fundamento en esquemas colaborativos y con tres líneas de acción definidas: (1) infraestructura tecnológica, consistente en dotar de hardware y conectividad a través de programas como Computadores para Educar y Compartel; (2) contenidos de calidad, buscando que se creen y se compartan contenidos de calidad dentro de la comunidad educativa a través de la plataforma Colombia Aprende; y (3) uso y apropiación de las TIC, dirigido a formar docentes en el uso y aprovechamiento de las TIC en la educación.

De esta manera es posible observar que en Colombia se está impulsando una incorporación de las TIC a la educación para transformar el modelo educativo. Este proceso ha hecho gran énfasis en la adopción de metodologías colaborativas: un ejemplo de esto es la plataforma Colombia Aprende como una comunidad de aprendizaje donde se busca de hacer del trabajo escolar e investigativo más "[...] creativo, abierto, plural, interactivo, ágil, colaborativo, interdisciplinario, multicultural, variado, agradable y potente $[\ldots]^{\prime 24}$. De esta forma, se puede observar la promoción del aprendizaje colaborativo para transformar el proceso educativo a través de la incorporación de las Tic.

Por lo tanto, las TIC abren una nueva serie de posibilidades en la educación y de cambios dirigidos a preparar a los nuevos aprendices del siglo XIX y a responder realidades de la sociedad actual. Dichas posibilidades hacen que el proceso educativo se transforme, y de esta forma aparecen nuevos escenarios educativos que antiguamente no se presentaban e incluso no se habían pensado en un mundo analógico. La realidad de la educación está cambiando y dejando de ser un modelo impartidor de conocimiento para ser un modelo de interacción, colaboración y creación de conocimiento.

\section{TECNOLOGÍAS DE LA INFORMACIÓN Y LA COMUNICACIÓN}

\section{EN LA EDUCACIÓN Y EXCEPCIONES AL DERECHO DE AUTOR}

Como se mencionó, una de las razones por las cuales se establecieron las excepciones y limitaciones en principio tanto en el ámbito nacional como en el internacional fue la necesidad de promover intereses públicos como la educación. No obstante, la realidad muestra que en la era actual las excepciones y limitaciones de la legislación colombiana no se están adaptando a lo que se está convirtiendo en el nuevo paradigma de educación. 
Colombia se encuentra en proceso de incorporar las TIC en la educación y de transformar su sistema educativo, como ya se ha señalado, y este proceso ha estado en marcha durante varios años y se han invertido grandes sumas de dinero; no obstante, se ha dejado de lado el análisis legal que debe acompañar este tipo de modelo en materia de derechos de autor. Las excepciones y limitaciones para la educación no fueron pensadas inicialmente para un escenario educativo fundamentado en las TIC, y de esta manera al intentar utilizar estas herramientas legales para promover la educación empoderada por el uso de las Tic se presentan principalmente tres inconvenientes: (1) dificultad para aplicar las excepciones y limitaciones al mundo digital; (2) dificultades para aplicar las excepciones y limitaciones a actores diferentes a los docentes y (3) dificultades para aplicar las excepciones y limitaciones a las actividades o metodologías colaborativas, propias de un modelo educativo transformado, problemas que se abordarán a continuación.

\section{A. LA APLICACIÓN DE LAS EXCEPCIONES Y LIMITACIONES AL MUNDO DIGITAL}

La aplicación de las excepciones y limitaciones al mundo digital establecidas en la ley colombiana es un asunto que ha generado muchas controversias e interrogantes en la doctrina ${ }^{25}$ por diversas razones. En primer lugar, existen dudas sobre si al aplicar las limitaciones y excepciones pensadas para el mundo analógico al mundo digital se cumpliría con la regla de los tres pasos ${ }^{26}$. En segundo lugar, algunas limitaciones no pueden ser aplicables al mundo digital ya que sus requisitos son relevantes solo para las tecnologías analógicas ${ }^{27}$. Finalmente, algunas limitaciones y excepciones no cubren todos los derechos que están involucrados en la realización de una transmisión digital ${ }^{28}$. Estos interrogantes todavía no han sido abordados por los jueces, y, por lo tanto, todavía no existen guías sobre su aplicación.

La situación de las limitaciones y excepciones en favor de la educación no es inmune a este escenario general. Las tres anteriores controversias se pueden observar en los artículos 22(b) y 22(c) de la Decisión 351 y 32, 38, 37 y 44 de la Ley 23 de 1982. El artículo 22(b) de la Decisión 351, como se explicó, permite realizar copias de calidad de materiales pertinentes sobre temas tratados en las clases y compartirlas con los alumnos o utilizarlos con fines de evaluación. No obstante, a pesar de la importancia de esta limitación para el ambiente educativo, dicha excepción no parece ser aplicable o trasladable al mundo digital.

25 Rengifo Ernesto, Un Nuevo reto del derecho en la edad de la información, 12 Revista la Propiedad Inmaterial, n. ${ }^{\circ}$ 12, 2008, 109,114, p. 105, disponible en http://revistas.uexternado. edu.co/index.php?journal=propin\&page=article\&op=view\&path\%5B\%5D=626.

26 Monroy Rodríguez Juan Carlos, Necesidad de nuevas limitaciones o excepciones para facilitar la digitalizacion y puesta a disposición de las obras protegidas en el marco de la educación virtual. Revista la Propiedad Inmaterial, n. ${ }^{\circ}$ 14, 2010, 195-208, p. 203, disponible en http://revistas.uexternado.edu.co/index.php?journal=propin\&page=article \&op=view \&path\% 5B\%5D=2479\&path\%5B\%5D=2115.

27 Monroy, supra note 8 , at 143 .

28 Id at 146 . 
El texto del mencionado artículo requiere que la reproducción objeto de la excepción se haga por medios reprográficos, lo cual significa que la excepción determina específicamente el tipo de tecnología que se puede utilizar para realizar la reproducción, esto es, la reprografía. La Dirección Nacional de Derecho de Autor (DNDA) define reproducción reprográfica de la siguiente manera:

[L]a reproducción reprográfica en materia de derecho de autor se entiende como todo sistema o técnica que permite realizar la reproducción facsimilar (fotografía, fotocopia, microfilme, etc.) de ejemplares de escritos y de obras gráficas en cualquier tamaño o forma y en su sentido estricto no resulta aplicable al entorno digital ${ }^{29}$.

Por lo tanto, cualquier otro tipo de reproducción que no por medio de tecnología reprográfica no estaría cubierto por esta excepción, excluyendo así la digitalización $^{30}$. La DNDA, siguiendo estos lineamientos normativos, ha conceptuado que escanear extractos de una obra legalmente adquirida y posteriormente subirlos a una plataforma virtual para fines educativos no está cubierto por la excepción de reproducción, ya que es claro que la reproducción debe ser por medios reprográficos ${ }^{31}$.

De igual manera, el artículo 32 de la Ley 23 de 1982 establece una excepción para la ilustración en la educación. No obstante, la aplicación de dicha excepción al mundo digital ha generado opiniones contrarias. Por un lado, algunos comentaristas, haciendo un análisis del texto de la ley, han concluido que dicha excepción no es aplicable a la educación virtual, por no cubrir dentro de su ámbito de aplicación el derecho de reproducción y comunicación público, ya que los términos "publicación” y "comunicación" utilizados por la disposición permitirían la comunicación pública de la obra mas no la reproducción, dejando de esta manera por fuera de su cobertura uno de los derechos exclusivos presentes durante una transmisión digital ${ }^{32}$.

Por otro lado, algunos comentaristas, interpretando el sentido de la excepción de ilustración incluida en el Convenio de Berna, la cual inspira la excepción incorporada en la legislación colombiana, afirman que la palabra "utilización", esgrimida en el Convenio al igual que en la ley colombiana, permite incluir dentro del ámbito de la excepción tanto el derecho de reproducción como el derecho de comunicación pública, y, consecuentemente, podría permitir la aplicación de dicha excepción al mundo virtual, dependiendo de cada caso ${ }^{33}$.

29 Dirección Nacional de Derecho de Autor, Competencia de la DNDA, radicación n. ${ }^{\circ}$ 1-2018-22353, p. 23, http://200.91.225.128/Intrane1/desarrollo/CONCEPTOSweB/arch_conceptos/12018-22353.pdf.

30 Monroy, supra note 8 at 143 .

31 Dirección Nacional de Derecho de Autor, Competencia de la DNDA, radicación n. ${ }^{\circ}$ 1-2010-10700, p. 3 http://200.91.225.128/Intrane1/desarrollo/CONCEPTOSWEB/arch_conceptos/2-2010-10700.pdf.

32 Rodríguez Moreno Sofía, La era digital y las excepciones y limitaciones al derecho de autor Bogotá, Universidad Externado de Colombia, 2004 p. 269-270.

33 Xalabarder, supra note 9, 14-19. 
No obstante, la DNDA parece estar un poco más inclinada a seguir la posición de no aplicabilidad, aunque por diferentes razones que llevan a analizar otro aspecto de la excepción que podría crear barreras para la aplicación de ésta al mundo digital. $\mathrm{Al}$ indagársele a la DNDA si cargar (upload) algunos extractos de libros legalmente adquiridos a una plataforma educativa virtual estaría cubierto por la excepción de la ilustración, la DNDA declaró que la plataforma virtual no puede ser considerada una obra, como la disposición requiere, y, por lo tanto, dicho actuar no está cubierto dentro de su ámbito de aplicación ${ }^{34}$. La DNDA, sin embargo, no se refirió a la no aplicabilidad de la excepción en el mundo digital en razón de la cobertura de los dos actos de reproducción y comunicación pública por la excepción, como lo ha discutido la doctrina.

Esta misma discusión se puede observar en la excepción y limitación en favor de las bibliotecas establecida en los artículos 22 (c) la Decisión 351 y 38 de la Ley 23 de 1982. Como se mencionó, bajo la legislación colombiana estas excepciones permiten a las bibliotecas reproducir una obra en un solo ejemplar con tres fines específicos: (1) para preservar el original y sustituirlo en caso de pérdida o destrucción; (2) para reemplazar las existencias permanentes de otra biblioteca en caso de pérdida o destrucción; y (3) para el uso de sus lectores. En consecuencia, cualquier otro tipo de reproducción con un propósito diferente no está cubierto ${ }^{35}$. De esta forma, aunque la reproducción para la conservación podría lograrse por medios digitales ${ }^{36}$, estas copias digitales no podían ser distribuidas ni comunicadas al público ya que estos derechos no están cubiertos por la excepción en favor de las bibliotecas. De igual manera, debido a que la excepción no cubre la reproducción para otros propósitos, la digitalización con el fin de crear una biblioteca digital no está cubierta bajo la excepción.

En el 2018, la Ley 1915 intentó dar cabida a la tecnología en bibliotecas, creando una nueva excepción para ellas. No obstante, dicha excepción, establecida en el artículo 16 (c), se quedó corta respecto a las posibilidades que ofrece la tecnología, permitiendo únicamente la puesta a disposición de los ejemplares de las obras lícitamente adquiridas en terminales instalados en las propias instalaciones de bibliotecas, archivos o centros de documentación. Lo anterior se reduce a permitir a dichas entidades poner a disposición de los usuarios a través de computadores los ejemplares de las bibliotecas para ser consultadas en la biblioteca misma. Esta excepción posiblemente ayuda respecto de problemas de disponibilidad de ejemplares en caso de obras altamente consultadas; no obstante, no permite crear bibliotecas digitales accesibles por fuera de las instalaciones de ellas.

34 Dirección Nacional de Derecho de Autor, Concepto, limitaciones y excepciones en la legislación colombiana, abril 9, 2010, radicación n. ${ }^{\circ}$ 2-2010-10700, p. 3, disponible en http://201.234.79.35/CONCEPTOSWEB/arch_conceptos/2-2010-10700.pdf.

35 Dirección Nacional de Derecho de Autor, supra note 12, at 3.

36 Rodríguez Moreno, supra note 33, at 272. 
Por último, del texto de la excepción de copia privada, establecido por el artículo 37 de la Ley 23 de 1982, y la excepción de uso privado, en el artículo 44 de la misma ley, se podría inferir que dichas excepciones son trasladables al entorno digital, ya que el texto legal no utiliza ninguna acepción para limitar el tipo de tecnología aplicable. No obstante, algunos comentaristas, al referirse a la aplicación de la excepción de copia y uso privado al mundo digital, han expresado su preocupación por que estas excepciones pueden no cumplir con la regla de los tres pasos, ya que las nuevas tecnologías hacen ampliamente disponibles varias copias de buena calidad fáciles de reproducir. De esta forma, bajo esta línea de pensamiento, permitir realizar copias sin restricción afectaría el mercado para la explotación de la obra protegida ${ }^{37}$.

\section{B. APLICACIÓN DE LAS EXCEPCIONES Y LIMITACIONES}

\section{A ACTORES DIFERENTES A LOS DOCENTES}

Además de las dudas sobre la aplicación de las excepciones y limitaciones al mundo digital, la utilización de éstas dentro del contexto de un proceso de educación basado en el uso de la tecnología genera otras preocupaciones. Lo anterior se debe a que las excepciones y limitaciones de la ley de derechos de autor para la educación fueron establecidas bajo un escenario tradicional de educación, donde en el proceso de enseñanza-aprendizaje solo interactuaban docentes y estudiantes y, entre éstos, el estudiante desempeñaba un papel pasivo.

Hoy en día la educación requiere el uso de las tecnologías, lo cual demanda un modelo pedagógico, como se explicó, basado en la colaboración, la interactividad y la creación de comunidades de aprendizaje, entre otras actividades. Esto tiene varias implicaciones sobre las excepciones y limitaciones puesto que bajo un modelo pedagógico colaborativo todos los actores de la comunidad de aprendizaje y el proceso de aprendizaje deberían estar cubiertos por las limitaciones y excepciones y tener derecho a hacer uso de los contenidos para poder realizar actividades educativas y que el proceso educativo fluya. No sería descabellado afirmar que ninguna persona estaría dispuesta a interactuar o colaborar en generación de contenidos o enseñanza si mediara la posibilidad de ser sancionados por una posible infracción. No obstante, actualmente la mayoría de excepciones y limitaciones bajo la legislación colombiana parece estar diseñada para cubrir el actuar de los profesores ${ }^{38}$.

Bajo la ley de derechos de autor de Colombia, por ejemplo, los estudiantes solo parecen beneficiarse de la excepción que permite la toma de notas y la ejecución de obras dentro de las instituciones educativas. No existen limitaciones o excepciones en favor del desarrollo del trabajo académico del estudiante. Una razón de la falta de excepción es que un uso académico de una obra protegida por un estudiante,

37 Barrero Piedad Lucía, Derecho de autor en ambientes virtuales, Bogotá, Universidad Cooperativa de Colombia, 2012, p. 69; Rodríguez Moreno, supra note 33, at 279-282.

38 Monroy, supra note 8 , at 64,72 . 
como lo explica Juan Carlos Monroy ${ }^{39}$, puede ser considerado un acto privado que no afecta los derechos de autor.

No obstante, bajo un modelo pedagógico colaborativo, se busca dar importancia a la labor académica del estudiante que mira hacia la generación de nuevos contenidos. Primero, a partir de aportes colaborativos entre todos los participantes se busca que los estudiantes compartan a través de herramientas Web 2.0 contenidos relevantes e información, lo cual significa que deben estar autorizadas la reproducción y la comunicación pública de materiales durante estas actividades; y, segundo, se busca incentivar la generación de materiales nuevos con base en contenidos preexistentes por medio de la transformación y la construcción sobre conocimiento previo, lo cual hace necesaria una autorización para la transformación. Por lo tanto, se convierte en un asunto de importancia la existencia de excepciones y limitaciones aplicables a los estudiantes. De lo contrario, un sistema educativo que busca darle un papel principal a la creación y transformación por parte del estudiante y la participación activa a intervinientes que no necesariamente estén actuando en un papel de maestros, como en el caso de las comunidades de aprendizaje, necesitaría actuar bajo licencias, que incrementan su costo y, por tanto, coartan la creación.

Bajo este nuevo tipo de metodologías, los estudiantes se deberían beneficiar principalmente de excepciones que les permitan reproducir y comunicar apartes de obras con motivos de aprendizaje, ya que las excepciones previstas actualmente que permiten realizar dicho tipo de actividades solo cobijan a profesores, tales como la excepción de ilustración para la educación y reproducción por medios reprográficos.

De igual manera, se necesitan excepciones y limitaciones que desvinculen los usos educativos de las instalaciones educativas. Con la importancia de las tecnologías nuevas actividades educativas se realizan por fuera del salón de clase y se trasladan a escenarios virtuales, pero la obligación de que algunas limitaciones se hagan dentro de instalaciones educativas no permite cobijar este nuevo tipo de actividades. Pensemos, por ejemplo, en el caso del docente Hernán López, del Colegio Marsella de la localidad de Kennedy, en Bogotá, quien por medio de Skype busca darles una visión global a sus estudiantes y conocer otras culturas, a través de charlas dictadas por expertos en otras partes el mundo ${ }^{40}$. No obstante, las excepciones y limitaciones en realidad no permitirían a sus estudiantes, por ejemplo, tomar apuntes de este tipo de conferencias, ya que la excepción exige que las conferencias sean dictadas en establecimientos de enseñanza.

Así mismo, posiblemente un profesor que esté dictando su clase por Zoom parece no estar autorizado para leer un capítulo de un libro o un poema completo a sus estudiantes, ya que la excepción de ilustración para la educación permite utilizar una obra como ilustración en la educación a través de emisión de radiodifusión.

$39 \mathrm{Id}$

40 El Tiempo, El profesor que viaja con sus alumnos por el mundo a través de Skype, El Tiempo 2019, disponible en https://www.eltiempo.com/vida/educacion/profesor-le-ensena-el-mundoa-sus-estudiantes-por-skype-390772. 
Esta se encuentra definida como "la transmisión, a los fines de su recepción por el público, de sonidos, o de imágenes y sonidos, por medios inalámbricos, ya sea por radio, televisión o satélite”¹ , definición que parece no incluir la transmisión digital.

Además, nuevas herramientas que se vuelven vitales para el aprendizaje hoy en día, como YouTube, y que son un ejemplo de comunidades de aprendizaje colaborativo, no se favorecerían de las excepciones y limitaciones, ya que estas han sido establecidas para un concepto de educación ejecutado en instituciones con una comunidad académica organizada ${ }^{42}$. ¿Quién no ha buscado aprender de algún tema en YouTube? incluso docentes de la educación formal utilizan esta herramienta para enseñar a sus estudiantes, mediante la grabación de un video o utilizando videos preexistentes. No obstante, al ser una plataforma pública y no ser parte de un aprendizaje formal, por ejemplo, no se podría hacer uso de extractos para la ilustración en la educación o reproducir una obra con motivos de enseñanza, en los términos de las excepciones y limitaciones.

\section{APLICACIÓN DE LAS EXCEPCIONES Y LIMITACIONES \\ A LAS ACTIVIDADES O METODOLOGÍAS COLABORATIVAS}

Los entornos colaborativos y comunidades de aprendizaje promueven no solo compartir contenidos en la red, sino también la transformación de contenidos por el uso de las Tic hacia la generación de nuevos contenidos. Como se explicó, en este nuevo tipo de aprendizaje se busca que los participantes interactúen colaborando, creando, editando y transformando conocimiento, con miras a la creación de nuevo conocimiento, a la innovación mediante el conocimiento preexistente. No obstante, bajo la legislación colombiana, las adaptaciones, modificaciones o transformaciones de una obra protegida no se encuentran cubiertas por ninguna excepción al derecho de autor.

Actualmente no hay limitaciones o excepciones previstas en la ley colombiana que cubran de manera amplia la transformación de las obras protegidas por el derecho de autor. Solamente hasta el 2018, con la Ley 1915, se creó la primera excepción al ejercicio del derecho exclusivo de transformación, permitiendo la realización de parodia y caricatura. Sin embargo, dicha excepción se queda corta respecto de transformaciones que no afectan la explotación de la obra original ni los intereses legítimos de los autores. Por el contrario, la ley de derechos de autor colombiana protege fuertemente los derechos de transformación o adaptación; por ejemplo, los autores de trabajos derivativos requieren el permiso del autor original de la obra para realizar la obra derivada y, de igual manera, necesitan autorización del autor de la obra original para luego explotar dicha obra derivada ${ }^{43}$.

41 омті, Principios Básicos del derecho de autor y derechos conexos, Ginebra, омpi, 2016, p. 12. 42 Monroy, supra note 8 , at 65 .

43 Dirección Nacional de Derecho de Autor, Concepto Generalidades, Obra Derivada (n. d.), radicación n. ${ }^{\circ} 1-2012-53648$ p. 5, disponible en http://201.234.79.35/CONCEPTOSWeb/ 
Por lo tanto, se puede observar que la actual ley de derechos de autor no está equipada para promover una fundamentación del modelo educativo con el uso de las tecnologías. Tal como se describió, existen diversas controversias acerca de si las excepciones y limitaciones podrían promover o permitir la realización de actividades de colaboración con el uso de herramientas Web 2.0. Lo anterior pondría en dificultades a estudiantes, maestros e investigadores que deseen adoptar un ambiente colaborativo y, como es la tendencia mundial, intentar incorporar las TIC en la educación, ya que tendrían la necesidad de adquirir una licencia para realizar actividades educativas bajo este sistema; de no ser así, enfrentarían las consecuencias que genera una infracción al derecho de autor.

\section{PROPUESTA DE NUEVAS EXCEPCIONES}

\section{A FAVOR DEL APRENDIZAJE COLABORATIVO}

Un aspecto novedoso que trajo consigo la Ley 1915 de 2018 fue el establecimiento en su artículo 17 de un proceso por medio del cual se busca actualizar las excepciones y limitaciones existentes cada tres años. La ley establece que se realizará una audiencia pública a cargo de la Dirección Nacional de Derecho de Autor, que será la entidad encargada de, en caso de considerarlo conveniente, presentar un proyecto de ley con miras a reformar, eliminar o crear nuevas excepciones y limitaciones al derecho de autor.

De esta manera, dado que la ley fue promulgada el 12 de julio de 2018, el próximo año se cumplirá el término establecido de tres años para programar la audiencia pública, con lo cual se abre una gran oportunidad para examinar asuntos como el aprendizaje colaborativo. Aunque la creación de excepciones encuentra muchos obstáculos dadas todas las obligaciones adquiridas en el ámbito internacional, en aras de abrir la discusión acerca de la necesidad de nuevas excepciones y limitaciones, $y$ en especial aquellas que faciliten un aprendizaje colaborativo virtual, la autora considera necesaria, entre otras, la creación de una nueva excepción que permita la transformación de obras, en adición a la modificación de algunas excepciones existentes para adaptarnos mejor a las oportunidades que brinda la tecnología.

Respecto de la nueva excepción para la transformación, buscará que la ley autorice sin necesidad de autorización o pago la transformación de una obra de tal manera que el mensaje de la nueva obra cambie, que estemos presentes ante un transformación de tal magnitud que el propósito de la nueva obra sea totalmente diferenciable de la primera, con nueva información y estética, y con nuevo entendimiento. Es decir, un cambio que vaya más allá de una simple parodia o sátira. Ahora bien: es también importante que la obra producto de la transformación no usurpe el mercado de la obra original, incluso el mercado de las obras derivadas, y 
esto se da porque simplemente el segundo uso es tan transformador que va incluso dirigido a otras personas objetivo, para las cuales no fue pensada la primera obra.

Así, dicha excepción de cierta maneja cobijaría lo que la casuística del fair use ${ }^{44}$ estadounidense ha buscado proteger: "el uso transformativo", que ha sido clave para la innovación. Este tipo de usos ha permitido el desarrollo, por ejemplo, del arte de apropiación, como Andy Warhol. Es menester resaltar que esta excepción también favorece a otro sector de los creadores, como el mencionado, y no solo a los usuarios, como suele pensarse acerca de las excepciones y limitaciones. Permitir este tipo de excepciones desde el punto de vista del aprendizaje colaborativo es clave, ya que la idea es que los estudiantes usen obras como materia prima para crear una obra propia y nueva.

Respecto de la excepción de toma de apuntes, es necesario eliminar el requisito de que dichas conferencias se dicten dentro de un establecimiento educativo, como requiere el artículo 40 de la Ley 23 de 1982, pero aun así mantener una directa relación con el proceso educativo, para evitar lesionar los intereses de los creadores. De igual manera, es importante que el artículo de reproducción para la enseñanza o evaluación no esté limitado solamente a reproducción reprográfica.

Este tipo de modificaciones, según ha mostrado la práctica, es conforme a los tratados internacionales y regionales firmados por Colombia. Por ejemplo, Perú, país también miembro de los principales tratados en derecho de autor y de la Comunicad Andina de Naciones, tiene presente en su legislación este tipo de excepciones. Respecto de la toma de apuntes, dicho país señala en el artículo 42 del Decreto Legislativo 822 de 1996 que "Las lecciones dictadas en público o en privado por los profesores de las universidades, institutos superiores y colegios podrán ser anotadas y recogidas en cualquier forma, por aquellos a quienes van dirigidas, pero nadie podrá divulgarlas o reproducirlas en colección completa o parcialmente, sin autorización previa y por escrito de los autores". De esta manera se pone énfasis en la calidad de la persona y no en el lugar donde se dictan las lecciones, y se permite utilizar mecanismos virtuales. No obstante, sería importante añadir dentro de la excepción también aquellas personas que ostentan de manera transitoria la calidad de docente tales como los conferencistas.

Respecto de la reproducción para la enseñanza y evaluación, el Perú realizó una modificación al Decreto Legislativo 822 de 1996 en el 2014, estableciendo la posibilidad de hacer reproducciones para la enseñanza por medios reprográficos y digitales, entre otros. El literal a del artículo 43 modificado por la Ley 30276 de 2014 estableció lo siguiente:

La reproducción por medio reprográfico, digital u otro similar para la enseñanza o la realización de exámenes en instituciones educativas, siempre que no haya fines de lucro y en la medida justificada por el objetivo perseguido, de artículos, discursos, 
frases originales, poemas unitarios, o de breves extractos de obras o del íntegro de obras aisladas de carácter plástico y fotográfico, lícitamente publicadas y a condición de que tal utilización se haga conforme a los usos honrados (cita obligatoria del autor) y que la misma no sea objeto de venta u otra transacción a título oneroso, ni tenga directa o indirectamente fines de lucro.

Así, se puede observar que de manera clara esta legislación acepta la reproducción para la enseñanza o evaluación por medios virtuales. Adoptar este tipo de excepción permitiría, en un aprendizaje colaborativo, facilitar la utilización de herramientas Web 2.0 para realizar actividades educativas e incluso aulas virtuales.

Finalmente, se debe pensar en la posibilidad de establecer alguna cláusula abierta aplicable específicamente a usos educativos que permita adaptarse a nuevos escenarios, especialmente aquellos donde no se está presente en un escenario formal o informal de educación, pero que son de gran utilidad para la innovación y el aprendizaje, por ejemplo algunos tutoriales dictados a través de redes sociales. Además, la excepción debe permitir actividades realizadas por estudiantes que hayan sido divulgadas. Ahora: es clave que dentro de la cláusula abierta se consagre como criterio la no afectación al mercado de los titulares.

\section{CONCLUSIÓN}

La tecnología y en particular la internet han empezado a influir y modelar la educación. Debido a la existencia de la tecnología, y gracias a ésta, se puede y debe empezar a pensar en un nuevo modelo educativo, con nuevas metodologías, como lo es la metodología colaborativa. Dicha metodología permite que todos los intervinientes del proceso educativo, esto es, los profesores y estudiantes, interactúen y colaboren para la creación de contenidos. De igual manera, la metodología colaborativa se abre a interactuar en comunidades de aprendizaje, donde los estudiantes y profesores pueden interactuar con investigadores, expertos, entre otros alrededor del mundo y de esta manera intercambiar experiencias, fuentes, materiales educativos y trabajar proyectos conjuntos. De esta manera, la metodología colaborativa lleva consigo la creación, transformación y edición de contenidos a través del uso de herramientas Web 2.0 como los blogs, los wikis, entre otros.

Colombia es uno de los países que están buscando transformar la educación a través de la tecnología, para lo cual ha invertido grandes cantidades de dinero en la ejecución de diversos programas y planes dirigidos a transformar el modelo educativo a través de las Tic, basados en esquemas colaborativos. No obstante, su ley de derechos de autor parece no estar adaptándose a dicha realidad, especialmente en el caso de excepciones y limitaciones.

La actual ley de derechos de autor establece unas excepciones y limitaciones en favor de la educación. Sin embargo, dichas excepciones y limitaciones fueron pensadas y establecidas en un escenario de educación tradicional en un mundo analógico. 
Intentar aplicar las actuales excepciones y limitaciones a un proceso educativo transformado genera controversias especialmente por tres motivos: (1) dificultad para aplicar las excepciones y limitaciones al mundo digital; (2) dificultades para aplicar las excepciones y limitaciones a los actores de un modelo educativo transformado y (3) dificultades para aplicar las excepciones y limitaciones a las actividades o metodologías colaborativas, propias de un modelo educativo transformado. Por tanto, la ley de derecho de autor colombiana debe ser modificada para promover o permitir la evolución de dicho modelo educativo.

De tal manera, se propone la creación de una excepción que permita la transformación, la modificación de las excepciones de reproducción para la evaluación y enseñanza y toma de apuntes y, finalmente, la creación de una cláusula abierta en beneficio de la educación.

\section{REFERENCIAS}

Anderson, J. ICT Transforming Education: a Regional Guide. Bangkok, Unesco, 2010. Recuperado de http://unesdoc.unesco.org/images/0018/001892/189216e.pdf.

Barrero, P. Derecho de autor en ambientes virtuales. Bogotá, Universidad Cooperativa de Colombia, 2012.

Dirección Nacional de Derechos de Autor (Dnda), Concepto: Competencia de la DNDA-Generalidades del Derecho de Autor-Objeto de protección del Derecho de Autor-El Alcance de las Facultades Exclusivas del Derecho de Autor- No Protección de las ideas- Titularidad del Derecho de Autor-Régimen de Transferencias-Limitaciones y Excepciones- Excepciones con fines de Enseñanza-Tramite conciliatorio-Acciones judiciales, radicado 1-2016-100918 (s. f.). Recuperado de http://200.91.225.128/Intrane1/desarrollo/CONCEPTOSWEB/encuentra_concepto.asp.

Dirección Nacional de Derechos de Autor (DNDA), Concepto: generalidades del derecho de autor y derechos conexos. Autorización previa y expresa-limitaciones, radicado 1-2014-27565, s. f. Recuperado de http://200.91.225.128/Intrane1/ desarrollo/CONCEPTOSWEB/arch_conceptos/1-2014-27565.pdf.

Dirección Nacional de Derechos de Autor, Concepto: limitaciones y excepciones en la legislación colombiana, radicado . $^{\circ} 1-2010-7340,2010$. Recuperado de http://200.91.225.128/Intrane1/desarrollo/CONCEPTOSWEB/arch_conceptos/1-2010-7340.pdf.

Dirección Nacional de Derechos de Autor, Concepto: Generalidades, obra derivada, radicado n. ${ }^{\circ} 1-2012-53648,2012$. Recuperado de http://200.91.225.128/ Intrane1/desarrollo/CONCEPTOSWEB/arch_conceptos/1-2012-53648.pdf.

KapCZynsK, A. Access to Knowledge: A Conceptual Genealogy. En Gaëlle Krikorian \& Amy Kapczynski (eds.) Access to Knowledge in the Age of Intellectual Property, New York: zone books, 2010, pp. 17-56. 
Kozma, R. B. A Framework for ICT Policies to Transform Education. En Unesco (Ed.), Transforming Education: The Power of ICT Policies París: Unesco, 2011, pp. 19-36. Recuperado de http://unesdoc.unesco.org/images/0021/002118/211842e.pdf.

Monroy, J. C., OMPI, Estudio sobre las limitaciones o excepciones al derecho de autory los derechos conexos en beneficio de las actividades educativas y de investigación en América Latina y el Caribe. Ginebra, Comité Permanente de Derecho de Autor y Derechos Conexos, 2009. Recuperado de http://www.wipo.int/meetings/en/ doc_details.jsp?doc_id=130303.

Monroy, J. C. Necesidad de nuevas limitaciones o excepciones para facilitar la digitalización y puesta a disposición de las obras protegidas en el marco de la educación virtual. La Propiedad Inmaterial, 14, 2010, 195-208.

Ministerio de Educación Nacional de Colombia. Programa Nacional de Nuevas Tecnologías. Colombia Aprende (s. f.). Recuperado de http://www. colombiaaprende.edu.co/html/home/1592/article-102549.html.

Ministerio de Educación Nacional de Colombia. www.colombiaaprend e.edu. co La nueva red del conocimiento. Al tablero, 29, 2004. Recuperado de http:// www.mined ucacion.gov.co/1621/article-87398.html.

Ministerio de TeCnologías de la Información y las Comunicaciones. Informe Rendición de Cuentas 2014, 2014. Recuperado de http://www.mintic.gov.co/ portal/604/articles-4323_recurso_1.pdf.

Ministerio de TeCnologías de la Información y las Comunicaciones. Informe de Gestión al Congreso de la República 2017, 2017. Recuperado de http://www. mintic.gov.co/portal/604/articles-54877_doc_pdf.pdf.

Palacio Puerta, M. Derechos de autor, tecnología y educación. Bogotá: Universidad Sergio Arboleda, 2016. Recuperado de: http://repository.usergioarboleda. edu.co/bitstream/handle/11232/692/Derechos\%20de\%20autor\%20web. pdf.?sequence $=1$.

Rengifo, E. Un nuevo reto del derecho en la edad de la información. Propiedad Inmaterial, 12, 2008, 105-120.

Ricketson, S. \& Ginsburg, J. International Copyright and Neighbouring Rights: The Berne Convention and Beyond, Oxford, Oxford University Press, 2nd ed., vol. I, 2006.

Rodríguez, S. La era digital y las excepciones y limitaciones al derecho de autor. Bogotá: Universidad Externado de Colombia, 2004.

SolAno, I. \& GutiÉrRez, I. Herramientas para la colaboración en la enseñanza superior: wikis y blogs. En Enseñanza Universitaria en el marco del Espacio Europeo de Educación Superior. Grupo de Investigación de Tecnología Educativa. Universidad de Murcia, 2007.

Unesco. Open and Distance Learning: Trends, Policy and Strategy Considerations. París, Unesco, 2002. Recuperado de http://unesdoc.unesco.org/images/0012/ 001284/128463e.pdf. 
Unesco. Technologies for Education. Potentials, parameters, and prospects. París: Unesco \& AED. 2002a. Recuperado de http://unesdoc.unesco.org/images/00 11/001191/11912 9e.pdf.

Unesco, Uso de TiC en la educación en América Latina y el Caribe, Montreal, Unesco. 2012. Recuperado de http://www.uis.unesco.org/Communication/Documents/ ict-regional-survey-lac-2012-sp.pdf.

WaHID, R. The Fairness of 'Stealing' Knowledge for Education. Journal of International Commercial Law and Technology, 6 (2), 2011, 86-95.

XALABARDER, R. OMPI, Estudio sobre las limitaciones y excepciones del derecho de autor para las actividades educativas en América del Norte, Europa, los paises Cáucaso, Asia Central e Israel, Ginebra. Comité Permanente de Derecho de Autor y Derechos Conexos, 2009. Recuperado de http://www.wipo.int/edocs/mdocs/ copyright/es/sccr_19/sccr_19_8.pdf. 